\title{
Aerosolized adenovirus-vectored vaccine as an alternative vaccine delivery method
}

\author{
Chad J Roy ${ }^{1}$, Alida Ault ${ }^{2 \dagger}$, Satheesh K Sivasubramani ${ }^{1 \dagger}$, J Patrick Gorres ${ }^{2}$, Chih-Jen Wei ${ }^{3}$, Hanne Andersen ${ }^{4}$, \\ Jason Gall ${ }^{5}$, Mario Roederer ${ }^{6}$ and Srinivas S Rao ${ }^{2^{*}}$
}

\begin{abstract}
Conventional parenteral injection of vaccines is limited in its ability to induce locally-produced immune responses in the respiratory tract, and has logistical disadvantages in widespread vaccine administration. Recent studies suggest that intranasal delivery or vaccination in the respiratory tract with recombinant viral vectors can enhance immunogenicity and protection against respiratory diseases such as influenza and tuberculosis, and can offer more broad-based generalized protection by eliciting durable mucosal immune responses. Controlled aerosolization is a method to minimize vaccine particle size and ensure delivery to the lower respiratory tract. Here, we characterize the dynamics of aerosolization and show the effects of vaccine concentration on particle size, vector viability, and the actual delivered dose of an aerosolized adenoviral vector. In addition, we demonstrate that aerosol delivery of a recombinant adenoviral vaccine encoding $\mathrm{H} 1 \mathrm{~N} 1$ hemagglutinin is immunogenic and protects ferrets against homologous viral challenge. Overall, aerosol delivery offers comparable protection to intramuscular injection, and represents an attractive vaccine delivery method for broad-based immunization campaigns.
\end{abstract}

\section{Introduction}

Conventional parenteral delivery of flu vaccines is limited in its ability to induce locally-produced immune responses in the respiratory tract as well as its capacity for efficient widespread distribution [1,2]. Recent studies evaluated intranasal delivery of recombinant vectorbased influenza vaccines as an alternative route of delivery that may enhance safety, efficacy, and ease of administration [3-6]. Vaccination in the respiratory tract may enhance protection against respiratory diseases such as influenza, tuberculosis, and measles, and may provide more generalized protection by inducing long-lasting mucosal immune responses $[7,8]$. Studies have also shown that mucosal immunity induced via intranasal delivery provides cross-protection against heterologous strains [9-15], and enhances heterosubtypic immunity for protection against multiple influenza A subtypes $[9,10,16,17]$. Other logistical advantages of an intranasal vaccine include the reduced risk of infection and contamination due to the non-use of needles and syringes,

\footnotetext{
*Correspondence: srao1@mail.nih.gov

+ Contributed equally

2Laboratory Animal Medicine, Vaccine Research Center, National Institutes of Health. Bethesda, MD 20895 USA

Full list of author information is available at the end of the article
}

and avoiding the need for disposal strategies of sharps after mass vaccination campaigns [18-20].

The currently licensed intranasal vaccine FluMist ${ }^{\mathrm{TM}}$ is a live-attenuated virus administered using a BectonDickenson AccuSpray ${ }^{\mathrm{TM}}$ device which generates a highspeed spray of large vaccine particles, with a mass median aerosol diameter $($ MMAD) $>70 \mu \mathrm{m}$. With particle size and speed being key factors determining aerosol deposition in the airway, these high-speed, large particle sprays are often trapped in the external nares and do not navigate to the internal airways which are the primary target of vaccination. Furthermore, droplets deposited in the nose can drip out or roll back toward the pharynx causing unpleasant sensations, diminishing acceptability of the vaccine [7]. In contrast, controlled aerosolization helps to minimize vaccine particle size variability and ensures delivery to the lower respiratory tract and internal target airways [21]. In animals, aerosol vaccination is currently used globally to immunize poultry against Newcastle disease and shows promise of successful immunization in fowls and pigs against a variety of diseases including fowlpox, infectious bronchitis, hog cholera, pseudorabies, erysipelas, gastroenteritis, pasteurellosis, and mycoplasmosis [19]. Notably, aerosol measles vaccination of 4 million Mexican schoolchildren 
in 1989-90 demonstrated a seroconversion rate of 52$64 \%$ (similar to subcutaneous administration) and an overall efficacy of $96 \%$, with excellent public acceptance and fewer side effects than subcutaneous vaccination [22]. However, while aerosol vaccination shows advantages in eliciting protective immune responses as well as cost-efficacy of administration, more studies are needed to further characterize the method and ensure that it is a safe and practical alternative.

Here, we elucidate the dynamics of aerosolization by analyzing particle size, vector viability, and actual delivered dose of an aerosolized adenoviral vector. This vector has been previously used alone or in combination with DNA prime immunizations to protect against lethal influenza challenges in mice and ferrets $[23,24]$. In addition, we compare the efficacy of aerosol vaccination to intramuscular (IM) injection of this recombinant adenoviral (Ad) vaccine encoding seasonal H1N1 immunogens against homologous challenge in ferrets.

Results indicate that vaccine concentration influences aerosol size, viability, and actual delivered dose, and should be considered in designing an optimal aerosol vaccination regimen. Results from the influenza challenge study indicate that aerosol vaccination elicits humoral immune responses and protects against H1N1 influenza challenge. Furthermore, the use of aerosol as the modality of vaccination has previously shown minimal to no lasting pathology in the lung, and is comparable to IM injection in immunogenicity and protection.

\section{Materials and methods \\ PARI eFlow ${ }^{\circledR}$ nebulizer device}

The PARI eFlow ${ }^{\circledR}$ device is a portable, electronic aerosol platform developed primarily to deliver liquid pharmaceutical therapies in a clinical setting [25-27]. The PARI eFlow $^{\circledR}$ aerosol device generates aerosols via a laser drilled membrane that is actuated via a piezoelectric crystal which pumps liquid through the membrane at relatively high velocity. This device was utilized in this study to deliver a biologic-based vaccine to the respiratory tract.

\section{Particle size characterization}

Empty Ad vector solutions were diluted in final formulation buffer (FFB) at seven different log concentrations, ranging from $10^{5}$ to $10^{11}$ particle units $(\mathrm{PU}) / \mathrm{mL}$, in order to establish size distribution characteristics of the aerosolized particles produced by the PARI eFlow ${ }^{\circledR}$ device. Three samples of $1 \mathrm{~mL}$ aliquots of each Ad concentration were aerosolized into a small plexiglass chamber using the PARI eFlow ${ }^{\circledR}$ device. An automated particle sizer (Aerodynamic Particle Size Model 3321, TSI Instruments, St. Paul, MN) was used to sample the generated atmosphere within the chamber and produce size and relative distribution estimates.

\section{Adenoviral aerosol viability}

Effects of the aerosol generation using the PARI device upon the relative viability of the Ad vector were unknown prior to this study. In order to determine the effect of aerosolization on the viability of an Ad vector and estimate the actual inhaled dose, empty Ad vector solutions were diluted at seven different log concentrations (as done in the particle size characterization experiment). Three samples of $1 \mathrm{~mL}$ aliquots were aerosolized as above, and the resultant aerosol was collected into an All-Glass Impinger (AGI) (Ace Glass, Vineland, NJ) containing $10 \mathrm{~mL}$ phosphate-buffered saline (PBS). The liquid capture of aerosol particles within the impinger contained an air sample that represented the atmospheric concentration of the Ad generated by the aerosol device. Immunofluorescence assays were performed in triplicate on collected samples to measure infectious titers post-aerosolization [28]. A non-aerosolized control for each concentration was also included. The fluorescent forming unit (FFU) results from the sampled aerosols were directly compared with the FFU content of the "pre" aerosol starting concentrations (expressed in FFU/l liquid starting concentration) in order to determine viral efficiencies after aerosol generation. The unitless factor (viral efficiency factor; $F_{e}$ ), defined as a ratio of the starting concentration (FFU/l liquid inoculum) to the aerosol concentration (FFU/1 aerosol) was used to express the relative viability of the Ad in aerosol (Table 1). While the magnitude of this value does not have a direct biological interpretation, comparison across conditions would reveal differential viral loss.

\section{Construction of recombinant Ad5 vaccine encoding hemagglutinin (HA)}

An Ad5-based first-generation vaccine expressing HA was constructed as described previously [29]. HA immunogen was derived from the A/Brisbane/59/2007 H1N1 isolate, a strain used in 2008-2009 Flumist ${ }^{\mathrm{TM}}$ trivalent intranasal vaccine [30]. Briefly, PacI-linearized shuttle vectors containing the influenza immunogen were recombined with the right side of Ad5 genomic DNA carried in a cosmid by use of Cre recombinase (Novagen, Madison, Wis.). The resulting recombinant was ethanol precipitated, dissolved in Tris-EDTA, and transfected into HEK 293 cells. Recombinant Ads were observed based on plaque formation 10 to 14 days after transfection. Viruses were amplified, purified two times through a $\mathrm{CsCl}$ gradient, and stored in PBS plus $15 \%$ glycerol at $-20^{\circ} \mathrm{C}$. 
Table 1 Effect of aerosolization on Ad viability.

\begin{tabular}{|c|c|c|c|c|c|c|}
\hline \multirow[b]{2}{*}{$\mathrm{C}_{\mathrm{s}}(\mathrm{FFU} / \mathrm{ml})$} & \multicolumn{2}{|c|}{ PS01 } & \multicolumn{2}{|c|}{ PS02 } & \multicolumn{2}{|c|}{ PS03 } \\
\hline & $\mathrm{C}_{\mathrm{a}}(F F U / \mathrm{ml})$ & $\mathrm{F}_{\mathrm{e}}$ & $\mathrm{C}_{\mathrm{a}}(F F U / \mathrm{ml})$ & $F_{e}$ & $\mathrm{C}_{\mathrm{a}}(F F U / \mathrm{ml})$ & $F_{e}$ \\
\hline $1.00 \mathrm{E}+08$ & $1.76 \mathrm{E}+02$ & $1.76 \mathrm{E}-06$ & n.d. & n.d. & $5.64 \mathrm{E}+01$ & $5.64 \mathrm{E}-07$ \\
\hline $1.00 E+09$ & $2.74 \mathrm{E}+02$ & $2.74 \mathrm{E}-07$ & $5.04 \mathrm{E}+02$ & $5.04 \mathrm{E}-07$ & $9.05 E+02$ & $9.05 \mathrm{E}-07$ \\
\hline $1.00 \mathrm{E}+10$ & 4.77E+03 & 4.77E-07 & $1.22 \mathrm{E}+04$ & $1.22 \mathrm{E}-06$ & $9.55 \mathrm{E}+03$ & $9.55 \mathrm{E}-07$ \\
\hline $1.00 \mathrm{E}+11$ & n.d. & n.d. & $6.25 E+04$ & $6.25 \mathrm{E}-07$ & $1.61 \mathrm{E}+05$ & $1.61 \mathrm{E}-06$ \\
\hline Mean(PS) \pm S.E. & \multicolumn{2}{|c|}{$8.37 \mathrm{E}-07 \pm 2.69 \mathrm{E}-07$} & \multicolumn{2}{|c|}{$7.84 \mathrm{E}-07 \pm 1.28 \mathrm{E}-07$} & \multicolumn{2}{|c|}{$1.01 \mathrm{E}-06 \pm 1.09 \mathrm{E}-07$} \\
\hline
\end{tabular}

Aerosol viability estimates for Adf.11D LN05224 from each independent experiment (PS01, PS02, PS03) using logarithmic starting concentrations ( $\mathrm{C}_{\mathrm{s}}$ ) and the corresponding aerosol concentration $\left(\mathrm{C}_{\mathrm{a}}\right)$ obtained from a proximity sampler located within a characterization chamber. The viral efficiency factor $\left(\mathrm{F}_{\mathrm{e}}\right)$, a unitless ratio of the $C_{s}$ and $C_{a}$, is used as an indicator of the dilution and associated loss of biologic activity during the process of aerosolization of the solution containing the adenovirus during characterization. The overall mean $\mathrm{F}_{\mathrm{e}}$ for all samples attempted was $8.8 \times 10^{-7}$.

\section{Ferret immunizations}

4-6 month old male, Fitch ferrets (Triple F Farms, Sayre, PA), sero-negative for exposure to currently circulating $\mathrm{H} 1 \mathrm{~N} 1, \mathrm{H} 3 \mathrm{~N} 2$, and B flu strains were housed and cared for at BIOQUAL, Inc. (Rockville, MD). These facilities are accredited by the American Association for the Accreditation of Laboratory Animal Care (AAALAC) International and meet NIH standards as set forth in the Guidelines for Care and Use of Laboratory Animals [31]. Prior to the start of the study, a temperature transponder (Biomedic Data Systems, Inc., Seaford, DE) was implanted into the neck of each ferret. Two groups of 7 ferrets were immunized twice at weeks 0 and 3 with an Ad5 vaccine encoding the HA gene from the A/Brisbane/59/2007 isolate at a dose of $1 \times 10^{10} \mathrm{PU}$. One group of ferrets received the Ad5 vaccine via IM injections in the upper thigh muscle while another group received the same vaccine via aerosol delivery using a PARI eFlow ${ }^{\circledR}$ nebulizer (Starnberg, Germany). Control ferrets were also immunized with empty Ad vectors via aerosol delivery.

For aerosol administration, ferrets were anesthetized with IM injections of $5-10 \mathrm{mg} / \mathrm{kg}$ ketamine and $0.5-1.0$ $\mathrm{mg} / \mathrm{kg}$ xylazine, and placed in a BSL-2 biosafety cabinet. With the PARI eFlow ${ }^{\circledR}$ device set up according to manufacturer's instructions, $1 \mathrm{~mL}$ of the vaccine was poured into the medication reservoir, and a modified facemask was applied to the ferret, ensuring a seal. The device was then activated and automatically deactivated after all of the liquid volume containing the vaccine was expended. The facemask was applied for an additional minute to ensure inhalation of any remaining aerosol.

\section{Hemagglutination inhibition ( $\mathrm{HI})$ assay}

Sera were treated with receptor-destroying enzyme (RDE) by diluting one part serum with three parts enzyme and incubated overnight in a $37^{\circ} \mathrm{C}$ water bath. The enzyme was inactivated by a $30 \mathrm{~min}$. incubation at $56^{\circ} \mathrm{C}$ followed by addition of six parts PBS for a final dilution of $1 / 10$. HI assays were performed in V-bottom 96-well plates using four hemagglutinating units (HAU) of virus and $1 \%$ horse erythrocytes as previously described [32]. Ferret sera were tested against homologous H1N1 influenza strain A/Brisbane/59/2007.

\section{Production of pseudotyped lentiviral vectors and measurement of neutralizing antibodies}

Production of pseudotyped lentiviral vectors for $\mathrm{H} 1 \mathrm{~N} 1$ and neutralization of pseudotyped viruses were performed as previously described [33].

\section{Ferret challenge experiments}

Ferrets were challenged three weeks after their second immunization with $\sim 10^{5} 50 \%$ Egg Infectious Dose $\left(\mathrm{EID}_{50}\right)$ of the H1N1 influenza strain A/South Dakota/6/ 2007 which is $99 \%$ homologous to A/Brisbane/59/2007. This virus had been expanded in 10-day old chicken eggs at BIOQUAL (Rockville, MD) from a seed stock obtained from the CDC (Atlanta, GA). The diluted virus stock was inoculated intranasally (in a volume of 0.25 $\mathrm{ml}$ per nostril) into ferrets that had been anesthetized with ketamine/xylazine. Ferrets were observed for clinical signs twice daily while weight and temperature measurements were recorded once daily by technicians blind to treatment groups. Ferrets that lost more than 25\% body weight or displayed severe clinical signs of infection (e.g. extreme lethargy or neurological impairment) were euthanized during the study. Remaining ferrets were euthanized on day 14 post challenge. Challenge experiments were performed in BSL-2 conditions and conducted at BIOQUAL, Inc. laboratories, which are approved for use by the USDA and CDC.

Nasal washes were obtained pre-challenge and on days 2,5 , and 7 post-challenge, and viral titers were determined using a real-time PCR assay as previously described [34]. Briefly, RNA was isolated from nasal washes and RT-PCR was performed using TaqMan reagents (Applied Biosystems, Foster City, CA) along with primers and probes covering a highly conserved region within the nucleoprotein gene. The detection range for this assay is $20-10^{7}$ copies $/ \mathrm{ml}$. Viral load data were analyzed using Tukey's Honestly Significant 
Difference (HSD) method to determine differences between immunized groups and controls. For each analysis, $\mathrm{P}$ values $<0.05$ were considered statistically significant.

\section{Results}

\section{Particle size characterization}

Characterization of the aerosolized Ad particle size was performed using a time-of-flight aerodynamic particle sizer (APS 3321, TSI Inc.). Proximity sampling was performed using the APS in a 16 liter plexiglass chamber. Resulting particle size distributions (Figure 1) showed MMADs fluctuated according to the relative concentration of the Ad present in the liquid carrier solution; the estimated MMAD ranged from 2.8 up to $6.5 \mu \mathrm{m}$, with relatively smaller MMAD ranges (3.1-2.8 $\mu \mathrm{m})$ in generation events that incorporated higher Ad concentrations $\left(10^{10}-10^{11}\right)$. The shifting MMAD is highlighted by a relatively stable geometric standard deviation (1.5-1.8) across all Ad concentrations used, indicating a minimal heterodispersity in the size distributions generated.
Particle size limitations for respirability in the ferret is unknown, but the majority of mass represented in most of the size distributions shown would be considered respirable in species of similar size, weight, and anatomy [35].

\section{Viability of aerosolized adenoviral vectors}

Results show the mean viral efficiency factor was relatively consistent over all concentrations, suggesting that the viability of the Ad will not be dramatically affected by the starting vector dose. Notably, the efficiency for this virus is not unusually low relative to the method of generation. A predictive dose delivered via inhalation can be derived from the mean viral efficiency factor, through estimation of the respiratory minute volume of the species, and the estimated aerosol concentration achieved with a particular viral starting concentration used. The nominal total titer administered to the ferrets was measured at $1.0 \mathrm{E}+10 \mathrm{PFU}$; the corresponding inhaled doses for the species by applying the viral efficiency factor estimate was 1.14E+04 PFU/animal.

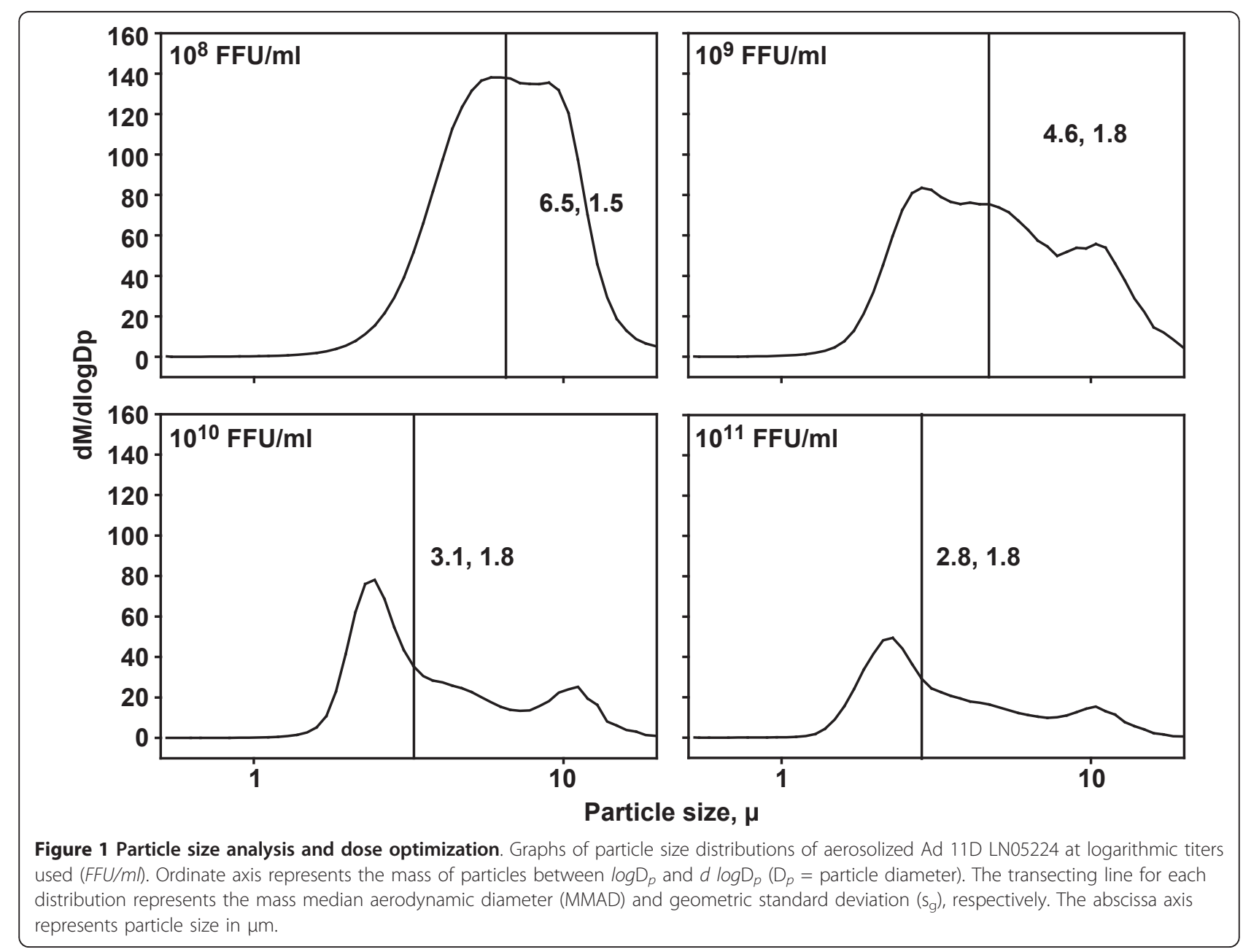


Table 2 Antibody titers of ferrets immunized with Ad5 encoding H1N1 HA.

\begin{tabular}{lcccc}
\hline & HI Assay (GMT) & Pseudotype Assay (IC50) \\
\hline & NC & Brisbane & NC & Brisbane \\
\hline Ad5-Control: AE ${ }^{\mathbf{a}}$ & $<20$ & $<20$ & - & - \\
\hline Ad5-Brisbane (×2): AE $^{\mathbf{a}}$ & 33 & 215 & 1064 & 2045 \\
\hline Ad-Brisbane (×2): IM & 98 & 390 & 1593 & 1805 \\
\hline $\mathrm{a}$ & & & \\
$\mathrm{H}=7$ \\
three weeks after the second immunization, against H1N1 strains of A/ \\
Brisbane/59/2007 and A/New Caledonia/20/99.
\end{tabular}

\section{Antibody responses in ferrets immunized with Ad5} encoding $\mathrm{HA}$

To compare the immune responses elicited by aerosol delivery to those by intramuscular injection, HI and pseudotype assays were performed on sera collected three weeks after the two immunizations by either route. As shown in Table 2, IM injection elicited moderately higher responses than aerosol delivery against both homologous and heterologous $\mathrm{H} 1 \mathrm{~N} 1$ viruses according to HI assays, although these differences were not found to be statistically significant via analysis by an unpaired t-test. Concurrently, the pseudotype inhibition assay also indicated that aerosol delivery elicited similar neutralizing responses as IM injection.

\section{Viral load reduction in ferrets challenged with $\mathrm{H} 1 \mathrm{~N} 1 \mathrm{~A} /$ South Dakota/2007}

To assess the extent of viral replication in the upper respiratory tract after challenge, nasal washes were performed at multiple time points following challenge and virus was quantified by RT-PCR (Figure 2). Significant reduction of viral loads was observed in aerosol-immunized groups compared to controls by day 2 post-challenge $(\mathrm{pc})(P<0.013)$. By day $5 \mathrm{pc}$, both immunization methods had significantly reduced viral loads compared to controls, $(P<0.001$ for AE, $P=0.007$ for IM). The reduction of viral loads was more prominent on day 7 pc as both immunized groups reached the minimum detection level (20 copies/mL nasal wash) while the virus persisted in the control group.

\section{Discussion}

Aerosol delivery is a potential alternative to parenteral injection for vaccine administration and may be

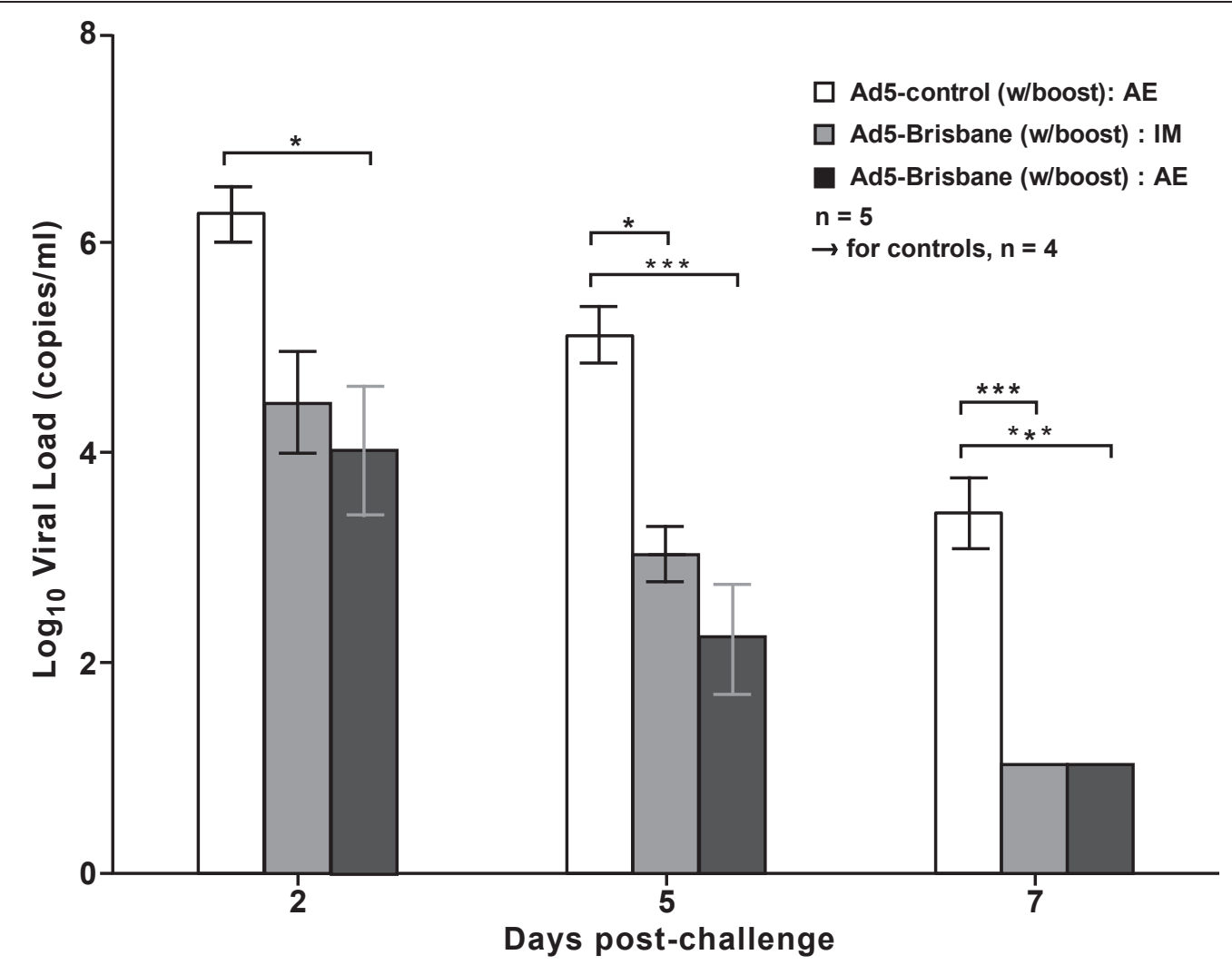

Figure 2 Viral load reduction in H1N1 A/South Dakota/06/2007 challenged ferrets. Viral loads were measured in H1N1-challenged ferrets at days 2,5 and 7 post-challenge. Bars indicate mean values of the $\log _{10}$ viral load, while error bars indicate standard error. Statistical differences were analyzed using Tukey's Honestly Significant Difference (HSD) method, and are indicated by asterisks where * represents a $p$-value between 0.05 and $0.01,{ }^{* *}$ indicates $0.01-0.001$, and ${ }^{* * *}$ indicates $<0.001$. Error bars indicate standard error. 
advantageous in eliciting robust immune responses at the site of infection against respiratory pathogens such as influenza $[7,8]$. Here we characterize important factors for optimizing such delivery including particle size, vector viability, and actual delivered dose of an aerosolized Ad vector. We also compare the efficacy of aerosol vaccination to IM injection against a seasonal H1N1 challenge in ferrets.

Results from the particle size characterization experiment suggest that the concentration of the Ad solution has an effect on the size of the particle generated. This is important since particle size affects the site of deposition in the respiratory tract which will then influence biological responses [35]. The respiratory system and prevailing anatomy of the ferret is considered susceptible to particle sizes consistent with respirability estimates in mammals such as dogs and cats in contrast to similarly sized rodent species [36,37]. High concentrations of Ad resulted in generally smaller aerosolized particles despite the nebulizer's design to produce nominal $4 \mu \mathrm{m}$ particles. This may be due to a clogging effect in which Ad precipitates on the nebulizing membrane, leaving residues which may hamper the efficiency of aerosolization. This hypothesis is supported by the observation that at high concentrations, the process of aerosolization took considerably longer than at low concentrations despite frequent changes and cleaning of nebulizing membranes. While other studies have similarly shown the effect of concentration on aerosol size [38], membrane "clogging" has not been previously documented and should be evaluated more closely.

Results from the seasonal influenza challenge study suggest that both aerosol and IM delivery of our Ad5 vaccine elicit similar levels of influenza-specific antibody responses, and are also comparable in reducing viral replication in the lungs of challenged ferrets. This is consistent with a previous study where we demonstrated robust immunogenicity and protection against lethal H5N1 influenza challenges in the ferret model [8] and is further supported by other recent challenge studies in mice $[7,14]$. While aerosolization may be comparable to IM delivery in serum antibody responses and viral load reduction, it may have significant advantages. One such advantage is the induction of local mucosal immune responses which may contribute to broader protection against heterologous strains and subtypes $[4,7,8]$, whereas parenteral vaccination is incapable of inducing these types of responses [1]. In addition, aerosol delivery may enhance acceptability of vaccination since it does not require needles and causes less discomfort than intranasal sprays [7]. Lastly, aerosol delivery is a safe, reliable, and economically feasible vaccine delivery platform that does not have the attendant safety risks of injections.

\section{Acknowledgements}

The authors would like to thank Mythreyi Shastri, Brenda Hartman, and Hana Bao for their assistance in manuscript preparation, and Linda Bessacque for administrative support.

\section{Author details}

${ }^{1}$ Infectious Disease Aerobiology, Division of Microbiology, Tulane National Primate Research Center, Covington, LA 70447 USA. ${ }^{2}$ Laboratory Animal Medicine, Vaccine Research Center, National Institutes of Health. Bethesda, MD 20895 USA. ํVector Core Section, Vaccine Research Center, National Institutes of Health, Bethesda, MD 20895 USA. ${ }^{4}$ BIOQUAL, Inc., Rockville, MD 20850 USA. ${ }^{5}$ GenVec Inc. Gaithersburg, MD 20878 USA. ImmunoTechnology Section, Vaccine Research Center, National Institutes of Health, Bethesda, MD 20895 USA.

\section{Authors' contributions}

CJR participated in the design of the study and analysis of the data. AA participated in the coordination and conduct of the study. SKS conducted the study and analyzed the data. JPG conducted the study and drafted the manuscript. CJW, HA, and JG carried out the immunoassays. MR and SSR conceived of the study and analyzed the data. All authors read and approved the final manuscript.

\section{Competing interests}

The authors declare that they have no competing interests.

Received: 6 September 2011 Accepted: 21 November 2011 Published: 21 November 2011

\section{References}

1. Brokstad KA, Eriksson JC, Cox RJ, et al: Parenteral vaccination against influenza does not induce a local antigen-specific immune response in the nasal mucosa. J Infect Dis 2002, 185(7):878-84.

2. Smith DJ, Bot S, Dellamary L, Bot A: Evaluation of novel aerosol formulations designed for mucosal vaccination against influenza virus. Vaccine 2003, 21(21-22):2805-12.

3. Imamura $\mathrm{T}$, Oshitani $\mathrm{H}$ : Mucosal immunity against influenza induced by attenuated recombinant Sendai virus. Expert Rev Vaccines 2011, 10(10):1393-5.

4. Park KS, Lee J, Ahn SS, et al: Mucosal immunity induced by adenovirusbased H5N1 HPAl vaccine confers protection against a lethal H5N2 avian influenza virus challenge. Virology 2009, 395(2):182-9.

5. Svindland SC, Jul-Larsen A, Pathirana R, et al: The mucosal and systemic immune responses elicited by a chitosan-adjuvanted intranasal influenza H5N1 vaccine. Influenza Other Respi Viruses 2011.

6. Quinn K, Quirion MR, Lo CY, Misplon JA, Epstein SL, Chiorini JA: Intranasal Administration of Adeno-associated Virus Type 12 (AAV12) Leads to Transduction of the Nasal Epithelia and Can Initiate Transgene-specific Immune Response. Mol Ther 2011.

7. Smith JH, Brooks P, Johnson S, et al: Aerosol vaccination induces robust protective immunity to homologous and heterologous influenza infection in mice. Vaccine 2011, 29(14):2568-75.

8. Song $\mathrm{K}$, Bolton DL, Wei CJ, et al: Genetic immunization in the lung induces potent local and systemic immune responses. Proc Natl Acad Sci USA 2010, 107(51):22213-8.

9. Epstein SL, Lo CY, Misplon JA, et al: Mechanisms of heterosubtypic immunity to lethal influenza A virus infection in fully immunocompetent, T cell-depleted, beta2-microglobulin-deficient, and J chain-deficient mice. J Immunol 1997, 158(3):1222-30.

10. Liang S, Mozdzanowska K, Palladino G, Gerhard W: Heterosubtypic immunity to influenza type $A$ virus in mice. Effector mechanisms and their longevity. J Immunol 1994, 152(4):1653-61.

11. Meitin CA, Bender BS, Small PA Jr: Influenza immunization: intranasal live vaccinia recombinant contrasted with parenteral inactivated vaccine. Vaccine 1991, 9(10):751-6. 
12. Tamura S, Ito $Y$, Asanuma $H$, et al: Cross-protection against influenza virus infection afforded by trivalent inactivated vaccines inoculated intranasally with cholera toxin B subunit. J Immunol 1992, 149(3):981-8.

13. Armerding D, Rossiter $H$, Ghazzouli I, Liehl E: Evaluation of live and inactivated influenza A virus vaccines in a mouse model. J Infect Dis 1982, 145(3):320-30.

14. Perrone LA, Ahmad A, Veguilla $V$, et al: Intranasal vaccination with 1918 influenza virus-like particles protects mice and ferrets from lethal 1918 and H5N1 influenza virus challenge. J Virol 2009.

15. Tumpey TM, Renshaw M, Clements JD, Katz JM: Mucosal delivery of inactivated influenza vaccine induces B-cell-dependent heterosubtypic cross-protection against lethal influenza A H5N1 virus infection. J Virol 2001, 75(11):5141-50

16. Nguyen $\mathrm{HH}$, Moldoveanu Z, Novak MJ, et al: Heterosubtypic immunity to lethal influenza $A$ virus infection is associated with virus-specific CD8(+) cytotoxic T lymphocyte responses induced in mucosa-associated tissues. Virology 1999, 254(1):50-60.

17. Song SK, Moldoveanu Z, Nguyen $\mathrm{HH}$, et al: Intranasal immunization with influenza virus and Korean mistletoe lectin C (KML-C) induces heterosubtypic immunity in mice. Vaccine 2007, 25(34):6359-66.

18. Laube BL: The expanding role of aerosols in systemic drug delivery, gene therapy, and vaccination. Respir Care 2005, 50(9):1161-76.

19. Roth Y, Chapnik JS, Cole P: Feasibility of aerosol vaccination in humans. Ann Otol Rhinol Laryngol 2003, 112(3):264-70.

20. Swayne DE: Avian influenza vaccines and therapies for poultry. Comp Immunol Microbiol Infect Dis 2008.

21. Hubbard RC, Casolaro MA, Mitchell M, et al: Fate of aerosolized recombinant DNA-produced alpha 1-antitrypsin: use of the epithelial surface of the lower respiratory tract to administer proteins of therapeutic importance. Proc Natl Acad Sci USA 1989, 86(2):680-4.

22. Cutts FT, Clements CJ, Bennett JV: Alternative routes of measles immunization: a review. Biologicals 1997, 25(3):323-38.

23. Rao SS, Kong WP, Wei CJ, et al: Comparative efficacy of hemagglutinin, nucleoprotein, and matrix 2 protein gene-based vaccination against H5N1 influenza in mouse and ferret. PLOS ONE 2010, 5(3):e9812.

24. Wei CJ, Boyington JC, MCTamney PM, et al: Induction of Broadly Neutralizing H1N1 Influenza Antibodies by Vaccination. Science 2010.

25. Guy EL, Bosomworth M, Denton M, Conway SP, Brownlee KG, Lee TW: Serum tobramycin levels following delivery of tobramycin (Tobi) via eFlow advanced nebuliser in children with cystic fibrosis. J Cyst Fibros 2010, 9(4):292-5.

26. Hubert D, Leroy S, Nove-Josserand $R$, et al: Pharmacokinetics and safety of tobramycin administered by the PARI eFlow rapid nebulizer in cystic fibrosis. J Cyst Fibros 2009, 8(5):332-7.

27. Parkins MD, Elborn JS: Aztreonam lysine: a novel inhalational antibiotic for cystic fibrosis. Expert Rev Respir Med 2010, 4(4):435-44.

28. Gall JG, Lizonova A, EttyReddy D, et al: Rescue and production of vaccine and therapeutic adenovirus vectors expressing inhibitory transgenes. Mol Biotechnol 2007, 35(3):263-73.

29. Aoki K, Barker C, Danthinne X, Imperiale MJ, Nabel GJ: Efficient generation of recombinant adenoviral vectors by Cre-lox recombination in vitro. Mol Med 1999, 5(4):224-31.

30. Fiore AE, Shay DK, Broder $K$, et al: Prevention and control of seasonal influenza with vaccines: recommendations of the Advisory Committee on Immunization Practices (ACIP), 2009. MMWR Recomm Rep 2009, 58(RR8):1-52.

31. National Research Council: Guide for the care and use of laboratory animals. Washington (DC), National Academies Press; 1996, Ref Type: Generic.

32. Stephenson I, Wood JM, Nicholson KG, Charlett A, Zambon MC: Detection of anti-H5 responses in human sera by $\mathrm{HI}$ using horse erythrocytes following MF59-adjuvanted influenza A/Duck/Singapore/97 vaccine. Virus Res 2004, 103(1-2):91-5.

33. Wei CJ, Boyington JC, Dai K, et al: Cross-neutralization of 1918 and 2009 influenza viruses: role of glycans in viral evolution and vaccine design. Sci Transl Med 2010, 2(24):24ra21.

34. Laddy DJ, Yan J, Kutzler M, et al: Heterosubtypic protection against pathogenic human and avian influenza viruses via in vivo electroporation of synthetic consensus DNA antigens. PLOS ONE 2008, 3(6):e2517.
35. Schlesinger RB: Comparative deposition of inhaled aerosols in experimental animals and humans: a review. J Toxicol Environ Health 1985, 15(2):197-214.

36. Lednicky JA, Hamilton SB, Tuttle RS, Sosna WA, Daniels DE, Swayne DE: Ferrets develop fatal influenza after inhaling small particle aerosols of highly pathogenic avian influenza virus A/Vietnam/1203/2004 (H5N1). Virol J 2010, 7:231.

37. Reznik GK: Comparative anatomy, physiology, and function of the upper respiratory tract. Environ Health Perspect 1990, 85:171-6.

38. Li X, LiU Q, Bi X, et al: An in vitro model to evaluate virus aerosol characteristics using a GFP-expressing adenovirus. J Med Microbiol 2008, 57(Pt 11):1335-9.

doi:10.1186/1465-9921-12-153

Cite this article as: Roy et al:: Aerosolized adenovirus-vectored vaccine as an alternative vaccine delivery method. Respiratory Research 2011 12:153.

\section{Submit your next manuscript to BioMed Central and take full advantage of:}

- Convenient online submission

- Thorough peer review

- No space constraints or color figure charges

- Immediate publication on acceptance

- Inclusion in PubMed, CAS, Scopus and Google Scholar

- Research which is freely available for redistribution 